\title{
Hemodiafiltration and plasma levels of axitinib in a patient with metastatic renal clear cell carcinoma
}

\author{
Jindrich Kopecky ${ }^{\mathrm{a}}$, Alena Ticha ${ }^{\mathrm{b}}$, Hana Janeckova ${ }^{\mathrm{c}, \mathrm{d}},{ }^{\text {, Bohuslav Melichara,e }}$
}

\begin{abstract}
Background. The standard treatment for metastatic renal cancer is based on vascular endothelial growth factor (VEGF) and mammalian target of rapamycin (mTor) inhibitors. Compared to other advanced tumors, the treatment of renal cancer is highly affected by impaired renal function; therefore, patients with severe renal insufficiency, including patients on hemodialysis, are generally excluded from clinical trials.

Case report. In the present manuscript we present the case of a renal cancer patient who underwent bilateral nephrectomy and received two lines of treatment. We hypothesized that axitinib, a tyrosine kinase inhibitor, would have a similar plasma concentration to patients without hemodialysis and that the levels before and after hemodiafiltration will not differ significantly, as observed in other targeted therapies.

Conclusion. The observed axitinib concentrations were at least an order of magnitude lower than expected based on the literature and measurements in other patients. The present case report indicates a potential risk of axitinib underdosing in patients on hemodiafiltration with the standard dose; therefore, drug dosage may need to be corrected based on the plasma levels of axitinib.
\end{abstract}

Key words: axitinib, renal cancer, chronic kidney failure, hemodialysis, protein-tyrosine kinases, nephrectomy

Received: December 10, 2017; Accepted with revision: April 13, 2018; Available online: May 15, 2018

https://doi.org/10.5507/bp.2018.021

${ }^{a}$ Department of Clinical Oncology and Radiotherapy, University Hospital Hradec Kralove and Faculty of Medicine in Hradec Kralove, Charles University, Czech Republic

${ }^{b}$ Department of Research and Development, University Hospital Hradec Kralove, Czech Republic

'Laboratory for Inherited Metabolic Disorders, Department of Clinical Biochemistry, University Hospital Olomouc, Czech Republic

${ }^{d}$ Institute of Molecular and Translational Medicine, Faculty of Medicine and Dentistry, Palacky University Olomouc, Czech Republic

eDepartment of Oncology, Faculty of Medicine and Dentistry, Palacky University Olomouc, Czech Republic

Corresponding author: Jindrich Kopecky, e-mail: kopecjin@fnhk.cz

\section{INTRODUCTION}

Renal cell carcinoma (RCC) accounts for 2-3\% of all adult malignant tumors, with Czech Republic presenting the highest RCC incidence and mortality rates ${ }^{1}$. Surgery is often the primary treatment for RCC; however, approximately one third of patients present locally advanced or metastatic diseaseat the time of the diagnosis. The standard treatment for metastatic RCC (mRCC), in addition to cytoreductive nephrectomy or the less common metastasectomy, is currently based on the vascular endothelial growth factor (VEGF) antibody bevacizumab, tyrosine kinase inhibitors (TKI) targeting VEGF receptors such as sorafenib, sunitinib, pazopanib, cabozantinib, lenvatinib and axitinib, mammalian target of rapamycin (mTOR) inhibitors temsirolimus and everolimus, cytokines (interferon- $\alpha$ and interleukin-2) or nivolumab. The sequential administration of active agents has transformed mRCC into a chronic disorder ${ }^{2}$.

Compared with other advanced tumors, the proportion of patients with renal impairment and/or chronic renal failure is particularly high in $\mathrm{mRCC}$ due to age, comorbidities, and a high proportion of patients with prior nephrectomy or even bilateral nephrectomy, in some cases the patients are dependent on hemodialysis for life. mRCC patients with severe renal failure, in particular those on hemodialysis, pose a therapeutic challenge to medical oncologists, as the registration trials of targeted therapy did not include patients with severe renal dysfunction.

To the best of our knowledge, there are currently no guidelines regarding the administration of targeted therapy and toxicity management in patients undergoing dialysis. In this report, we present the case of a patient who underwent bilateral nephrectomy for RCC, received axitinib in the second line and its plasmatic levels monitored while on hemodiafiltration.

\section{CASE REPORT}

A 67-year-old man was first diagnosed in July 2011 with right (pT2N0M0, grade 2) and in July 2014 with left (pT3aN0M0, grade 4) kidney clear cell carcinoma; nephrectomy was performed in both cases. In the latter event, the surgery was an emergency procedure due to massive bleeding with bladder tamponade. Five months after left nephrectomy, tumor relapse in the original site of left kidney and multiple metastatic lung nodules were diagnosed. The medical history of the patient is notable for arterial hypertension (treated with amlodipine) and an earlier episode of acute toxic hepatitis due to alcohol 
Table 1. Basic laboratory parameters before and after axitinib therapy.

\begin{tabular}{|c|c|c|}
\hline Parameter [unit (physiological range)] & Initial values & Final values \\
\hline White blood cell [109/L (4-10)] & 6.29 & 8.18 \\
\hline Red blood cell[ $\left[10^{12} / \mathrm{L}(4-5.8)\right]$ & 3.30 & 3.11 \\
\hline Hemoglobin[g/L (135-175)] & 98.00 & 100.00 \\
\hline Thrombocytes[109/L (150-400)] & 164.00 & 129.00 \\
\hline ALT $[\mu \mathrm{kat} / \mathrm{L}(0-0.83)]$ & 0.11 & 0.22 \\
\hline AST $[\mu \mathrm{kat} / \mathrm{L}(0-0.83)]$ & 0.23 & 0.27 \\
\hline GMT [ $\mu \mathrm{kat} / \mathrm{L}(0.13-1.02)]$ & 0.63 & 0.53 \\
\hline Urea[mmol/L (2.8-8.1)] & 7.20 & 15.50 \\
\hline Kreatinin $[\mu \mathrm{mol} / \mathrm{L}(62-106)]$ & 366.00 & 701.00 \\
\hline $\mathrm{TSH}[\mathrm{mU} / \mathrm{L}(0.27-4.2)]$ & 1.73 & 2.48 \\
\hline Albumin[g/L (35-52)] & 39.60 & 43.20 \\
\hline Total protein $[\mathrm{g} / \mathrm{L}(64-83)]$ & 64.40 & 68.70 \\
\hline
\end{tabular}

abuse, although the patient had abstained from alcohol since 2005. After the second nephrectomy, regular to dialysis had to be started. Laboratory analysis showed no pathology except normocytic anemia (see Table 1), and the patient was in good performance status (ECOG 1). According to the MSKCC scoring system from 2002, the patient was stratified as intermediate risk, and treatment with sunitinib at dose of $37.5 \mathrm{mg}$ was started on 29 January 2015 with good tolerance. After 4 months of treatment, lung progression was determined according to the RECIST 1.1 criteria, and second-line treatment with axitinib was started on 11 June 2015 at $5 \mathrm{mg}$ BID. The treatment was well tolerated, with fatigue and stable normocytic anemia being the only side effects. Radiologic evaluation 3 months later revealed progressive disease in lung and retroperitoneal lymph nodes. Because of his good performance status, the patient was offered a third line of treatment which he refused, and only supportive care could be provided.

\section{METHODS}

In the present case, an FX CorDiax 600 High-Flux Hemodiafilterwith effective surface area $1.6 \mathrm{~m}^{2}$ (Fresenius Medical Care) was used in the treatment; hemodiafiltration was done thrice weekly, starting at 6:45 am $(+/-15 \mathrm{~min})$, the treatment duration was fixed at $300 \mathrm{~min}$. The blood flow (QB) was $380 \mathrm{~mL} / \mathrm{min}$ in average and the the dialysate flow (QD) was set automatically to be between $400-600 \mathrm{~mL} / \mathrm{min}$ with average flow of $500 \mathrm{~mL} / \mathrm{min}$ for most of the time during the procedure. The prescribed dose of filtration with its adequate substitution for whole time of hemodiafilration procedure was in average 28.4 $\mathrm{L} / 5 \mathrm{~h}(5680 \mathrm{~mL} / \mathrm{h})$. The quality of delivered dialysis was controled by the Kt/V ratio mesasured with help of OCM (On-line clearance monitoring), which was in avarage 2.1.

The patient received $0.8 \mathrm{~mL}$ of nadroparine calcium during the procedure and had transfusion twice during the treatment with axitinib. After two hours from beginning of dialysis session, the patient was regulary given diazepam (10 mg intravenously) because of restless legs syndrome.
The patient took axitinib regularly at $6 \mathrm{am}$, with a second dose at $6 \mathrm{pm}$. The compliance with the axitinib administration was checked routinely during the hemodiafiltration and also based on patient report. The first blood collection was done after 2 days on axitinib treatment.

As internal control we performed control measurements in two patients on axitinib ( $5 \mathrm{mg}$ BID) with preserved kidney function. Those two male patients were similar age (68 and 70) and the blood was collected 2, 4 and $5 \mathrm{~h}$ after drug intake.

Blood samples were collected into K3-EDTA vacutainer tubes (BD Diagnostics, Franklin Lakes, New Jersey) before and after hemodiafiltration (within the same time period) thrice weekly during the first month and twice weekly in the second month. Whole blood $(5 \mathrm{~mL})$ was centrifuged (Centrifuge 2702R; Eppendorf) at 1,500 x $g$ for $15 \mathrm{~min}$, after which plasma was removed and frozen at $-70{ }^{\circ} \mathrm{C}$ for future analysis of axitinib plasma levels.

Before the analysis of axitinib plasma levels, all frozen samples were thawed at $4{ }^{\circ} \mathrm{C}$, the plasma $(50 \mu \mathrm{L})$ was mixed with an internal standard $(50 \mu \mathrm{L} ; 10 \mathrm{ng} / \mathrm{mL}$ dasatinib-d 8 in methanol) and precipitated using $0.3 \mathrm{~mol} / \mathrm{L}$ zinc sulfate in $70 \%$ methanol $(150 \mu \mathrm{L})$. After vortex mixing and centrifugation ( $5 \mathrm{~min}, 14,500 \mathrm{x} \mathrm{g}$ ) the supernatant was analyzed by high-performance liquid chromatography (UltiMate 3000; Dionex, Sunnyvale, CA) coupled with tandem mass spectrometry (Triple Quad 6500; AB Sciex, Sunnyvale, CA) which was already validated for this kind of measurment eralier ${ }^{3}$.

The present study was performed according to the World Medical Association Declaration of Helsinki and the patients signed an informed consent for subsequent publication of the treatment results.

\section{RESULTS}

Table 2 presents the measured values in the plasma samples collected before and after hemodiafiltration during two months of axitinib therapy. Median plasma concentrationof axitinib before dialysis was significantly higher compared to the paired sample after dialysis (me- 
Table 2. Plasma concentrations of axitinib measured during hemodiafiltration (HD).

\begin{tabular}{lcccccccccccccccc}
\hline $\begin{array}{l}\text { Day of sample } \\
\text { colletion }\end{array}$ & $1 *$ & 4 & $8 *$ & 11 & 13 & 15 & 18 & 32 & 34 & 39 & 41 & 46 & 48 & 50 & 55 & 60 \\
\hline $\begin{array}{l}\text { Axitinib level before } \\
\text { HD (ng/mL) }\end{array}$ & 7.19 & 0.61 & 0.28 & 0.51 & 0.21 & 0.57 & 1.13 & 1.14 & 0.78 & 1.16 & 6.41 & 1.06 & 2.76 & 5.39 & 1.81 & 1.35 \\
$\begin{array}{l}\text { Axitinib level after } \\
\text { HD }(\mathrm{ng} / \mathrm{mL})\end{array}$ & 1.57 & 0.27 & 0.28 & 0.28 & 0.18 & 0.09 & 0.38 & 0.49 & 0.22 & 0.45 & 0.87 & 0.4 & 0.83 & 1.14 & 0.86 & 0.59 \\
\hline
\end{tabular}

* indicate the haemosubstitution

dian $1.14 \mathrm{ng} / \mathrm{mL}$ vs. $0.43 \mathrm{ng} / \mathrm{mL}$; Wilcoxon paired rank) $P<0.001$.

\section{DISCUSSION}

The data on the outcome of patients with renal insufficiency are limited to single case reports or small case series, and the data on patients on hemodialysis are rather sparse. Most pharmacokinetic evaluation data on patients with mRCC on hemodialysis are done for sunitinib, sorafenib, everolimus, with only one case report for axitinib ${ }^{4-7}$.

Axitinib is a small molecule tyrosine kinase inhibitor with antitumor activity in mRCC. Axitinib has a short effective plasma half-life (range, 2.5-6.1 h) and is absorbed relatively rapidly, reaching maximum observed plasma concentrations (Cmax) within $4 \mathrm{~h}$ of oral administration. At adose of $5 \mathrm{mg}$ twice per day, the geometric mean peak plasma concentration is $27.8 \mathrm{ng} / \mathrm{mL}$. Axitinib is highly (>99\%) bound to human plasma proteins, with preferential binding to albumin, and metabolized primarily in the liver by cytochrome P450 (CYP) 3A4/5, later eliminated through hepatobiliary excretion.

We hypothesized that axitinib, same as other TKIs, would have similar plasma levels before and after hemodiafiltration (as seen in hemodialysis), not differing too much from the expected values. Hemodialysis has not been significantly linked to modified plasma concentrations of sunitinib or its active metabolite, based on their comparable arterial and venous concentrations $2 \mathrm{~h}$ after the start of hemodialysis. However, plasma exposure to sunitinib and SU12662 was significantly lower in patients requiring hemodialysis compared with those with normal renal function ${ }^{8,9}$.

To our best knowledge, there is just only one case study of a patient on hemodialysis during treatment with axitinib $^{7}$, reporting that hemodialysis has no impact on axitinib plasma concentrations and pharmacokinetics. The performed tests also excluded the impact of the membranes used in hemodialysis on axitinib plasma concentrations.

Our data seem to be in contradiction to the published case report by Thiery-Vuillemin et al. ${ }^{7}$. Although the interval between administration of axitinib and the start of dialysis was similar to our case, our axitinib plasma concentrations were out of range; however, it is difficult to compare these two case reports because of the different study design.

In our case we used hemodiafiltration as an alternative to hemodialysis. This therapy combines conventional diffusive hemodialysis with the convectional component of hemofiltration. Thus hemodiafiltration offers not only small molecule clearance, it has the potential added benefits of improved larger molecule clearance. Fruther we were unable to perform pharmakocinetics calculations due to the unwillingness of our patient to provide blood samples at regular intervals during hemodiafiltraton or between hemodiafiltration sessions. Regardless, this is the first time that extremely low axitinib concentrations have been associated with hemodiafiltration. These low plasma levels could explain the clinical outcome of our patient presented as an aggressive disease with fast progression.

Except for hemodiafiltration, there could be some other hypothetical reasons for the low plasma concentration of axitinib. One of such could be extrapolated from the data on drugs predominantly or completely metabolized by the liver, indicating that patients with renal insufficiency may have impaired plasma protein binding of many drugs. This often results in a larger apparent volume of distribution and a larger clearance of the drug compared to that observed in patients with normal renal function. Hence, renal disease can also influence the pharmacokinetics of drugs that are eliminated from the body by biotransformation rather than by renal excretion. Repetitive administration of a given dose of a drug that is eliminated from the body by nonrenal mechanisms may result in considerably lower steady-state concentrations of total drug in the plasma of patients with renal disease than in that of patients with normal renal function. On the other hand, in the absence of liver disease, the average steady-state serum concentration of free (unbound) drug is likely to be the same in both groups of patients ${ }^{10}$.

Another reason for axitinib plasma alteration could be due to genetic polymorphisms of adenosine triphosphate-binding cassette $(\mathrm{ABC})$ transporters (ABSG2 and $\mathrm{ABCB} 1$ ), which are involved in the absorption and excretion of various drugs; several studies in this regard have demonstrated that such polymorphisms affect plasma concentrations of oral anticancer drugs ${ }^{11}$, and in this regard, the concentration of axitinb is higher in patients with ABCG2 a homozygous variant genotype than in those carrying a heterozygous or wild type variant ${ }^{12}$. Nevertheless, another study suggests that the adjustment 
of the axitinib dose based on the genotype of individual patients is not warranted because no statistically significant association between genetic polymorphisms and axitinib plasma exposure has been determined ${ }^{13}$. Despite the unavailability of data showing any clear correlation between genetic polymorphism and plasma concentrations of axitinib, it must be emphasized that the mechanisms underlying any individual differences in the plasma concentrations of oral molecular targeting agents are more complex and includes additional factors such as metabolic enzymes and co-administrated drugs.

To make the list complete, it should be added that coadministration of axitinib with strong CYP3A4/5 inducers (e.g., rifampin, dexamethasone, phenytoin, carbamazepine, rifabutin, and St. John's Wort) may decrease plasma concentrations ${ }^{14}$.

To minimize the bias caused by the measurement technique, we performed control measurements in two patients with preserved kidney function in order to compare the plasma levels of axitinib. In one case, we took the measurement 2 and $4 \mathrm{~h}$ after drug intake with a result of 7.71 and $13.4 \mathrm{ng} / \mathrm{mL}$, respectively; in the second case, the value was of $20.5 \mathrm{ng} / \mathrm{mL}$ after $5 \mathrm{~h}$ of drug intake. It is apparent that the levels observed in the present case on any occasion were at least an order of magnitude lower than reported or observed in the other two patients treated in our center.

The reason for the significant difference, between pre- and post-dialysis plasma levels, in our case could be partially caused by decrease plasma protein binding of axitinib in chronic renal insuficiency, increasing the free (unbound) fraction which might be then dialysed. Although chronic renal insuficieny increases plasma levels of $\alpha 1$-acid glycoprotein, when according to the product labeling, axitinib binds to $\alpha 1$-acid glycoprotein with moderate degree, one would predict increased binding of axitinib in renal insuficiency. However, in most cases drug binding is reduced or remains unchanged. The reduced binding of axitinib to $\alpha 1$-acid glycoprotein may result from reduced binding affinity and competing ligands circulating in the plasma of end-stage renal disease patients ${ }^{15}$. In our case such hypothetical competing ligand could be diazepam, which was given regularly during hemodialysis. Diazepam has high affinity to plasma albumin and can compete with axitinib, which is then removed by dialysis. Even if reported just in one case report as non-significant ${ }^{7}$, we could not exclude the influence of a potential adhesion of axitinib to the dialysis membrane.

Based on pharmacokinetic and pharmacodynamic studies, no axitinib dose adjustment is warranted based on age, ethnicity, body weight, or other covariates, including body surface area and creatinine clearance, ALT and AST activity, or bilirubin concentration ${ }^{16}$. However, it should be noted that in these studies, the lack of impact could be caused by enrollment bias as the patients in clinical trials are required to have normal or near-normal renal and hepatic function on study entry.

There is evidence of a relationship between axitinib plasma exposure and the probability of therapy response ${ }^{17}$; and, although anecdotal, the present case report indicates that the decreased exposure as a consequence of dialysis may have an unfavorable impact on treatment outcome. Among the currently used TKI, axitinib is peculiar because of adaptive dosing in its administration ${ }^{17,18}$. This approach, possibly guided by drug concentration, may be even more important in patients on hemodiafiltration.

Over the past years, new therapeutic approaches have been introduced, such as immunotherapy. Although there are no data on the use of nivolumab in patients on hemodialysis, based on the mechanism of drug action we can presume that there is only limited potential for pharmacokinetic interference with hemodiafiltration.

The present report has obvious limitations. First, only a single case was studied. Second, there might be slight differences in the plasma levels of drug between the samples taken from AV fistula and those collected from vein, although we tried to minimize this bias by frequent sampling. There is also a limitation in the design of the study, where it would be desirable to provide AUC of axitinib, but the patient consented to the collection of the blood sample only at the start and ends of hemodiafiltration session, and was not willing to be punctured repeatedly during or after the session.

\section{CONCLUSION}

Because there are no established guidelines regarding drug administration and toxicity management in mRCC patients undergoing dialysis, therapy should be administered with caution. In the present case, no unexpected toxicity and good tolerance was observed. Although it seems that hemodialysis itself does not have direct impact on axitinib pharmacokinetic, based on our data, hemodiafiltration as an unusual circumstance for treatment should be considered as a risk factor concerning underdosing. Therefore, it is advisable to monitor and correct the drug dosage based on the plasma levels of axitinib. Further confirmation on whether hemodiafiltration is linked with axitinib plasma alteration is needed on a larger cohort of patients.

Acknowledgement: The study was supported by the Charles University Faculty of Medicine in Hradec Kralove grant [SVV-2016-260286], [SVV-2017-260396], UK Progres [Q40/06]; the grant of Ministry of Health [MH CZ-DRO UHHK, 00179906]; the grant of Ministry of Education of Czech Republic [LO1304]; and the internal grant of Palacký University Olomouc [IGA-LF-2017-015]. Thanks also to Daniel Díaz, Ph.D. for his assistance in proofreading this manuscript.

Author contributions: JK study design, JK, BM paper preparation and data interpretation, AT, HJ blood analysis, interpretation and paper revision.

Conflict of interests statement: Jindrich Kopecky received honoraria for consultancy from BMS, Novartis and Pfizer; Bohuslav Melichar received honoraria for consultancy from BMS, Novartis, Pfizer, Astellas and Roche; 
Alena Ticha and Hana Janeckova declare that they have no conflict of interest.

\section{REFERENCES}

1. Buchler T, Klapka R, Melichar B, Brabec P, Dusek L, Vyzula $R, A b r a h a m o v a J$ J. Sunitinib followed by sorafenib or vice versa for metastatic renal cell carcinoma--data from the Czech registry. Ann Oncol 2012;23(2):395-401.

2. Nishida H, Fukuhara H, Yamagishi A, Sakurai T, Shibasaki T, Kawazoe $\mathrm{H}$, Kato T,Tomita Y. Sequential molecularly targeted drug therapy including axitinib for a patient with end-stage renal failure and metastatic renal cell carcinoma. Hemodial Int 2016;20(2):E1-4.

3. Bouchet S, Chauzit E, Ducint D, Castaing N, Canal-Raffin M, Moore N Titier K,Molimard M. Simultaneous determination of nine tyrosine kinase inhibitors by 96-well solid-phase extraction and ultra performance LC/MS-MS. Clin Chim Acta 2011;412(11-12):1060-7.

4. Izzedine $\mathrm{H}$, Etienne-Grimaldi M C, Renee N, Vignot S,Milano $\mathrm{G}$. Pharmacokinetics of sunitinib in hemodialysis. Ann Onco 2009;20(1):190-2.

5. Lunardi G, Vannozzi M O, Armirotti A, Nicodemo M, Venturin $M$, Cavallini L. Temsirolimus in patients with renal cancer on hemodialysis. J Clin Oncol 2008;26(34):5652-3; author reply 5653-4.

6. Thiery-Vuillemin A, Curtit E, Maurina T, Montange D, Succi C, Nguyen T, Kim S, Montcuquet P, Pivot X, Royer B. Hemodialysis does not affect everolimus pharmacokinetics: two cases of patients with metastatic renal cell cancer. Ann Oncol 2012;23(11):2992-3.

7. Thiery-Vuillemin A, Orillard E, Mouillet G, Calcagno F, Devillard N, Bouchet S,Royer B. Hemodialysis does not impact axitinib exposure: clinical case of a patient with metastatic renal cell carcinoma. Cancer Chemother Pharmacol 2017;79(6):1273-6.

8. Khosravan R, Toh M, Garrett M, La Fargue J, Ni G, Marbury T C, Swan $\mathrm{S}$ K, Lunde N M,Bello C L. Pharmacokinetics and safety of sunitinib malate in subjects with impaired renal function. J Clin Pharmacol 2010;50(4):472-81.
9. Khan G, Golshayan A, Elson P, Wood L, Garcia J, Bukowski R,Rini B. Sunitinib and sorafenib in metastatic renal cell carcinoma patients with renal insufficiency. Ann Oncol 2010;21(8):1618-22.

10. Gibaldi M. Drug distribution in renal failure. Am J Med 1977;62(4):471-4.

11. Mizuno T, Fukudo M, Terada T, Kamba T, Nakamura E, Ogawa O, Inui $\mathrm{K}$, Katsura T. Impact of genetic variation in breast cancer resistance protein (BCRP/ABCG2) on sunitinib pharmacokinetics. Drug Metab Pharmacokinet 2012;27(6):631-9.

12. Kato H, Sassa N, Miyazaki M, Takeuchi M, Asai M, Iwai A, Noda Y, Gotoh M,Yamada K. Association of axitinib plasma exposure and genetic polymorphisms of $A B C$ transporters with axitinib-induced toxicities in patients with renal cell carcinoma. Cancer Chemother Pharmacol 2016;78(4):855-62.

13. Brennan M, Williams J A, Chen Y, Tortorici M, Pithavala Y,Liu Y C. Meta-analysis of contribution of genetic polymorphisms in drugmetabolizing enzymes or transporters to axitinib pharmacokinetics. Eur J Clin Pharmacol 2012;68(5):645-55.

14. Rugo H S, Herbst R S, Liu G, Park J W, Kies M S, Steinfeldt H M, Pithavala Y K, Reich S D, Freddo J L,Wilding G. Phase I trial of the oral antiangiogenesis agent AG-013736 in patients with advanced solid tumors: pharmacokinetic and clinical results. J Clin Oncol 2005;23(24):5474-83.

15. Dreisbach A W ,Lertora J J. The effect of chronic renal failure on hepatic drug metabolism and drug disposition. Semin Dial 2003;16(1):45-50.

16. Garrett M, Poland B, Brennan M, Hee B, Pithavala Y K,Amantea M A. Population pharmacokinetic analysis of axitinib in healthy volunteers. Br J Clin Pharmacol 2014;77(3):480-92.

17. Rini B I, Melichar B, Fishman M N, Oya M, Pithavala Y K, Chen Y, Bair A H,Grunwald V. Axitinib dose titration: analyses of exposure, blood pressure and clinical response from a randomized phase II study in metastatic renal cell carcinoma. Ann Oncol 2015;26(7):1372-7.

18. Rini B I, Melichar B, Ueda T, Grunwald V, Fishman M N, Arranz J A, Bair A H, Pithavala Y K, Andrews G I, Pavlov D, Kim S,Jonasch E. Axitinib with or without dose titration for first-line metastatic renal-cell carcinoma: a randomised double-blind phase 2 trial. Lancet Oncol 2013;14(12):1233-42. 